\title{
Experiments on Free-to-Pivot Hover Motions of Flat Plates
}

\author{
Kenneth Granlund ${ }^{1}$ and Michael $\mathrm{OL}^{2}$ \\ U.S. Air Force Research Laboratory, Wright-Patterson Air Force Base, OH 45433, U.S.A. \\ Luis Bernal ${ }^{3}$ and Steve Kast ${ }^{4}$ \\ University of Michigan, Ann Arbor, MI
}

\begin{abstract}
Using force measurements and flow visualization in a water tunnel, we consider motions of a flat plate with square edges, free to pivot about its leading edge, between incidence angle limits of $\pm 45^{\circ}$. The plate's leading edge undergoes a prescribed sinusoidal motion, either of rectilinear translation, or of pivoting or waving about a fixed point 0.5 chords away from one of the plate's wingtips. During most of the translation semi-stroke in either direction, the plate rests against its incidence limiter to produce a positive angle of attack; this reverses on the opposite semi-stroke, producing a motion akin to normal-hover with delayed rotation. Two geometries are considered: a nominally 2D or wall-to-wall plate, and a plate of aspect ratio 3.4. Reynolds number effects in the range of 5000-20,000 were not found to be significant, but the ratio of stroke amplitude to plate chord determines Depending on stroke to chord amplitude, lag between plate rotation and translation will differ, and the resulting vortex production history and aerodynamic load production history will differ. Large stroke to chord ratios produces higher thrust coefficients and simpler vortex wakes. Thrust coefficient histories are very similar between the translating $2 D$ and $A R=3.4$ plates, and resistive force coefficients are very similar amongst all three cases suggesting that whatever distinctions between the three cases may be present in the flowfield due to putative spanwise pressure gradient, these effects do not systematically alter the integrated aerodynamic forces.
\end{abstract}

\section{Introduction}

A significant challenge for flapping-wing Micro Air Vehicles, especially in hover, is to remove all vestiges of fixed flight surfaces and to produce thrust, lift and control forces/moments strictly through modulation of flapping wing kinematics. This generally means minimizing the actuated degrees of freedom while relying on passive deflections to orient the flight surfaces in aerodynamically favorable positions. Doman et al.1, proposed a flight control scheme based on the flapping-wing configuration developed by Wood et al.2, where the wing leading edge is directly actuated in a sweeping motion, but the wing incidence angle is free to float between limiters. The incidence angle is generally right at the limiter throughout the "translation" phase of each half-stroke, with a rapid rotation from one limiter side to the other, at or near the extrema of each half-stroke. Doman et $\mathrm{al}^{1}$ assume in their analysis a quasisteady lift coefficient time history throughout the translation stroke, and a non-lifting rotation phase. The former assumption is probably justified for conceptual-design purposes based on results for sinusoidal periodic plunge $\left(\mathrm{Ol}\right.$ et al. $\left.{ }^{8}\right)$, where one finds remarkable robustness of the simple $\mathrm{C}_{\mathrm{L}}=2 \pi \alpha$ even for large incidence angles. But the latter assumption is only valid if the stroke fraction occupied by the rotation is small, and post-rotation transients dye out quickly. And, since the incidence angle time history during rotation is passively accepted from the combination of body dynamics (wing mass and moment of inertia, hinge dynamics, etc.) and aerodynamic loads (time history of pressure distribution on the wing), the actual incidence angle history is not known a priori. One may find various lags between rotation and translation, and asymmetries between commencement and completion of incidence angle change at each half-stroke extremum.

\footnotetext{
${ }^{1}$ Post-Doctoral Scholar, Air Vehicles Directorate

${ }^{2}$ Senior Aerospace Engineer, Air Vehicles Directorate, Michael.O1@wpafb.af.mil

${ }^{3}$ Associate Professor, Department of Aerospace Engineering.

${ }^{4}$ Student, Department of Aerospace Engineering.
} 
In this work, we seek to connect the fairly broad literature on "normal hover" with imposed translation and rotation time history (Milano and Gharib ${ }^{3}$, Kurtulus et al. ${ }^{4}$, Shyy et $\mathrm{al}^{5}$; with an upper bound of Reynolds number typically around 1000), with a rectilinear and nonrectilinear version of the motion assumed by Doman et $\mathrm{al}^{1}$. Using the speed and power of a large electric motion rig, and limiting ourselves to rigid plates, we study passive pitch change across $\pm 45^{\circ}$ incidence change, with prescribed sinusoidal motion of the leading edge, at Reynolds numbers from 5000 to 20,000, based on maximum leading edge speed. We can therefore compare with the more usual problem of normal hover ${ }^{6}$, where a $2 \mathrm{D}$ or finite aspect ratio plate undergoes imposed sinusoidal oscillation in both translation and rotation, with various phase differences between the two. We can also compare with the fixed incidence angle rotating plate experiments of Babinsky and Jones ${ }^{7}$, where the plate is wings about a fixed point near one of its tips. The overall question is to what extent the rectilinear and nonrectilinear motions differ in flowfield history and aerodynamic force production - the latter being the thrust produced and the force opposing the motion.

\section{Experimental Setup}

\section{Facility and Motion Mechanism}

The U.S. Air Force Research Laboratory's Horizontal Free-surface Water Tunnel is fitted with a three degree of freedom electric rig enabling independent control of pitch or rotation, plunge or heave, and "surge" or streamwise-aligned translation. In the present study, the tunnel is operated as a tank, with the oscillator rig providing the model motions. A photograph of the tunnel and two model installations are shown in Figure 1. More detail on the rig operation and discussion of experimental error is given in $\mathrm{Ol}$ et al. ${ }^{8}$, while the facility is discussed in $\mathrm{Ol}$ et al. ${ }^{9}$

The 3-component oscillator rig is controlled through a Galil DMC 4040 Ethernet controller. Pitch and plunge are via a pair of motors mounted vertically on a plate above the tunnel test section, shown in the middle portion of Figure 1. Each motor actuates a vertical "plunge rod", which connects via a bushing to a coupler piece. In prior work ${ }^{10}$, the coupler piece was imbedded in an airfoil or flat-plate model. This is acceptable for runs with nonzero free stream, where presumably interference from the plunge rods would result in separated flow structures convecting downstream. But with the water tunnel run as a tank, there is no convective relief from interferences, and therefore to better isolate the model from the rig, a cantilever mounting arrangement is used, depicted in the middle portion of Figure 1. For imposed-translation experiments, the nominally $2 \mathrm{D}$ is wall-to-wall with $1.0 \mathrm{~mm}$ gap on each tip, $5 \mathrm{~cm}$ chord, $6 \%$ thickness and rectangular edges. The $\mathrm{AR}=3.4$ plate has a span of $17 \mathrm{~cm}$, and is identically mounted and oscillated. The test section floor is $\sim 6$ chords below the plate trailing edge.

The upstream plunge rod of the pitch-plunge portion of the mechanism is constrained to move purely vertically, whereas the downstream plunge rod is allowed to pivot in the test section vertical plane of symmetry. The desired angle of incidence and vertical position time history of the model are converted to position commands for each linear motor. The pitch pivot point can be varied by suitable choice of phase and amplitude difference in trajectory of front or rear plunge rod. For all cases where the pitch pivot point is not coincident with the bushed end of the front plunge rod, there will be a parasitic streamwise displacement of the model, which would be unavoidable unless the front plunge rod were to be allowed to pivot similarly to the downstream one. This is removed using the third degree of freedom, surge, which also actuates the fore and aft translating motion of the model. Surge is achieved using a larger linear motor mounted horizontally aft of the pitch-plunge carriage, with 48 " peak-to-peak stroke and nominal speed up to $1 \mathrm{~m} / \mathrm{s}$.

In free-to-pivot imposed translations, the model is hinged at the midspan of its leading edge, and constrained to rotate within $\pm 45^{\circ}$ from its rest (vertical) position. As its support is translated, pressure difference between the advancing and retreating side of the plate forces the plate against one of its pitch limiters, effectively placing it at $45^{\circ}$ angle of attack. This is reversed in the reverse direction of 
translation. Only the surge motor of the motion rig is used, while the pitch/plunge motors are held fixed. In the flapping (waving) motion, the model is hinged from a bar along the leading edge, and the bar undergoes imposed sinuosoidal rotational motion of $\pm 90^{\circ}$ extent.

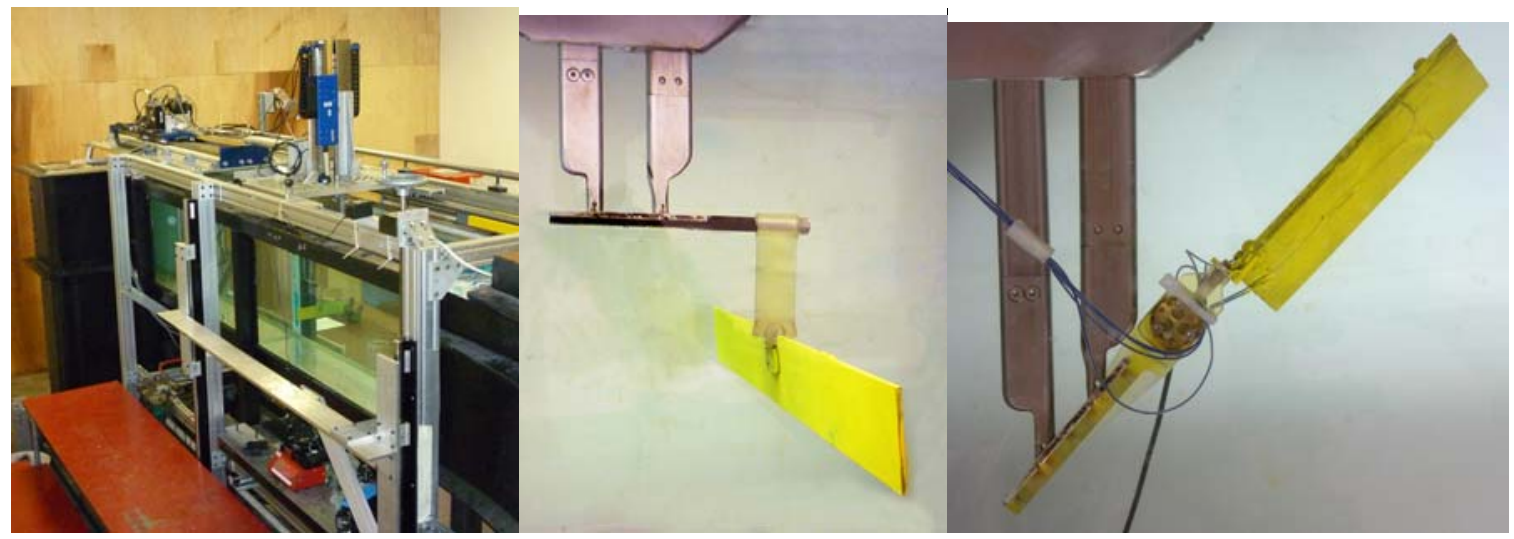

Figure 1. Test section and motion apparatus mounted above test section of the AFRL Horizontal Free-surface Water Tunnel (left); wall-to-wall plate set up for sinusoidal imposed fore-aft translation (middle) and AR=3.4 plate set up for flapping, in maximal upstroke position (right). The force balance is the metal cylinder in the right-hand image.

\section{Force Measurement and Flow Visualization}

For dye injection, a wand with $0.5 \mathrm{~mm}$ internal diameter, injecting concentrated blue food colouring, was glued to the model and exits near the hinged edge or the trailing edge, firing outboard along the span. Unfortunately for hover experiments dye injection is less successful than for motions with free-stream ${ }^{8}$, because large strain rates cause rapid dye dissipation, while running the dye at high flow rates introduces unacceptable disturbances of the ambient flow and large agglomerations of dye near the motion stroke endpoints.

Force measurements were with an ATI Nano-25 waterproof 6-component balance (right-hand image in Figure 1), oriented to align its maximally sensitive channels with the longitudinal forces and moments (lift, drag and pitching moment). For maximal force balance sensitivity and reduction of vibrations, the balance should be near the model's center of pressure. This however introduces unacceptable interference. The aerodynamically cleaner arrangement is an aft sting, where the model is mounted some distance ahead of the balance, and the balance is integrated into a housing that connects to the two vertical plunge rods. Here one must be careful about the stiffness of the sting and the balance itself. The "apparent mass" of the water accelerated along with the model - essentially a circular cylindrical slug of water with length the same as that of the model, and diameter equal to the model's chord - can be 10 times larger than that of the model, sting, and metric portion of the balance. Therefore, in direct contradistinction with wind tunnel unsteady aerodynamics testing practice, it is not necessary to build a light model or sting, and instead the preference is for high strength at the expense of mass.

\section{Results}

Two families of translational cases and one family of waving or flapping cases comprised the data set. Translations were for a wall-to-wall or nominally 2D plate (Table 1), and for a plate of aspect ratio 3.4 (Table 2). Flapping was limited to the plate of aspect ratio 3.4 (Table 3). 
Table 1. Translating wall-to-wall plate. Values of translation physical frequency, in Hz, along columns of constant stroke to chord ratio, result in a nominal Reynolds number (left column) based on peak translational speed. Values in grey indicate cases with dye injection images.

\begin{tabular}{rrrrrrr} 
Nominal Re & \multicolumn{7}{c}{ Stroke to chord ratio } & & & \\
& 6.20 & 3.10 & 1.55 & 1.16 & 0.775 & 0.3875 \\
4100 & & & & & & 1.064 \\
5200 & & & & & & 1.330 \\
6500 & 0.106 & 0.211 & 0.422 & 0.563 & 0.844 & 1.688 \\
7400 & & & & 0.634 & & \\
8300 & & & 0.532 & & 1.064 & 2.128 \\
9800 & 0.158 & 0.316 & & & & \\
10400 & & & 0.670 & & & \\
11600 & & & & 1.000 & & \\
13000 & 0.211 & 0.422 & 0.844 & 1.126 & 1.688 & \\
14700 & & & & 1.267 & & \\
16400 & 0.264 & 0.532 & 1.064 & & & \\
20600 & 0.333 & 0.665 & 1.330 & & &
\end{tabular}

Table 2. Translating aspect ratio 3.4 plate. Values of translation physical frequency, in Hz, along columns of constant stroke to chord ratio, result in a nominal Reynolds number (left column) based on peak translational speed. Values in grey indicate cases with dye injection images.

\begin{tabular}{rrrrrrrr} 
Nominal Re & \multicolumn{7}{c}{ Stroke to chord ratio } \\
& 6.20 & 3.10 & 1.55 & 1.16 & 0.775 & 0.3875 \\
& & & & & & \\
6500 & 0.106 & 0.211 & 0.422 & 0.563 & 0.844 & 1.688 \\
9800 & 0.158 & 0.316 & 0.634 & 0.844 & 1.267 & \\
10300 & & & & & 1.330 & \\
13000 & 0.211 & 0.422 & 0.844 & 1.126 & 1.688 & \\
20600 & 0.333 & 0.670 & 1.330 & & &
\end{tabular}

Table 3. Waving aspect ratio 3.4 plate. Motion frequency tabulated with nominal Reynolds number based on peak tip speed. Value in grey indicates dye injection case.

\begin{tabular}{lrrrrrr} 
& \multicolumn{7}{c}{$90^{\circ}$ deg peak-to-peak stroke in all cases } \\
frequency & 0.211 & 0.422 & 0.532 & 0.670 & 0.844 & 1.000 \\
$\operatorname{Re}$ & 6500 & 13000 & 16300 & 20600 & 26000 & 30800
\end{tabular}

\section{Wall-to-wall plate with sinusoidal rectilinear fore-aft motion}

We first consider a survey of reduced frequency/amplitude, while keeping Reynolds number constant. In the absence of a free-stream, educed frequency becomes a purely kinematic variable, and is here is taken as the ratio of stoke amplitude to plate chord. Figure 2 shows snapshots of dye injection for every 30 degrees of phase of fore-aft sinusoidal motion, for four cases: frequency of $0.211 \mathrm{~Hz}$ and amplitude of 3.1 chords; frequency of $0.422 \mathrm{~Hz}$ and amplitude of 1.55 chords; and frequency of $0.844 \mathrm{~Hz}$ and amplitude of 0.775 chords. The vertical purple lines in Figure 2 attempt to mark the extreme of the plate's leading edge. We note that in convective problems, where there is a free-stream, dye injection is a powerful surrogate for out-of-plane vorticity component, as rotational effects evidently dominate diffusion. But in hover, in the absence of a free stream, dye streaks diffuse quickly, and evidently a more quantitative approach such as particle mage velocimetry becomes imperative. Nevertheless, one can 
discern clear differences between the three cases not only in the vortex shedding phenomena, but also in the history of plate incidence angle. Frame-by-frame viewing of the dye injection videos allows for extraction of the motion kinematics: verification of the trajectory of the plate's leading edge, and recording of the plate's incidence angle as a function of phase. This is given in Figure 3.

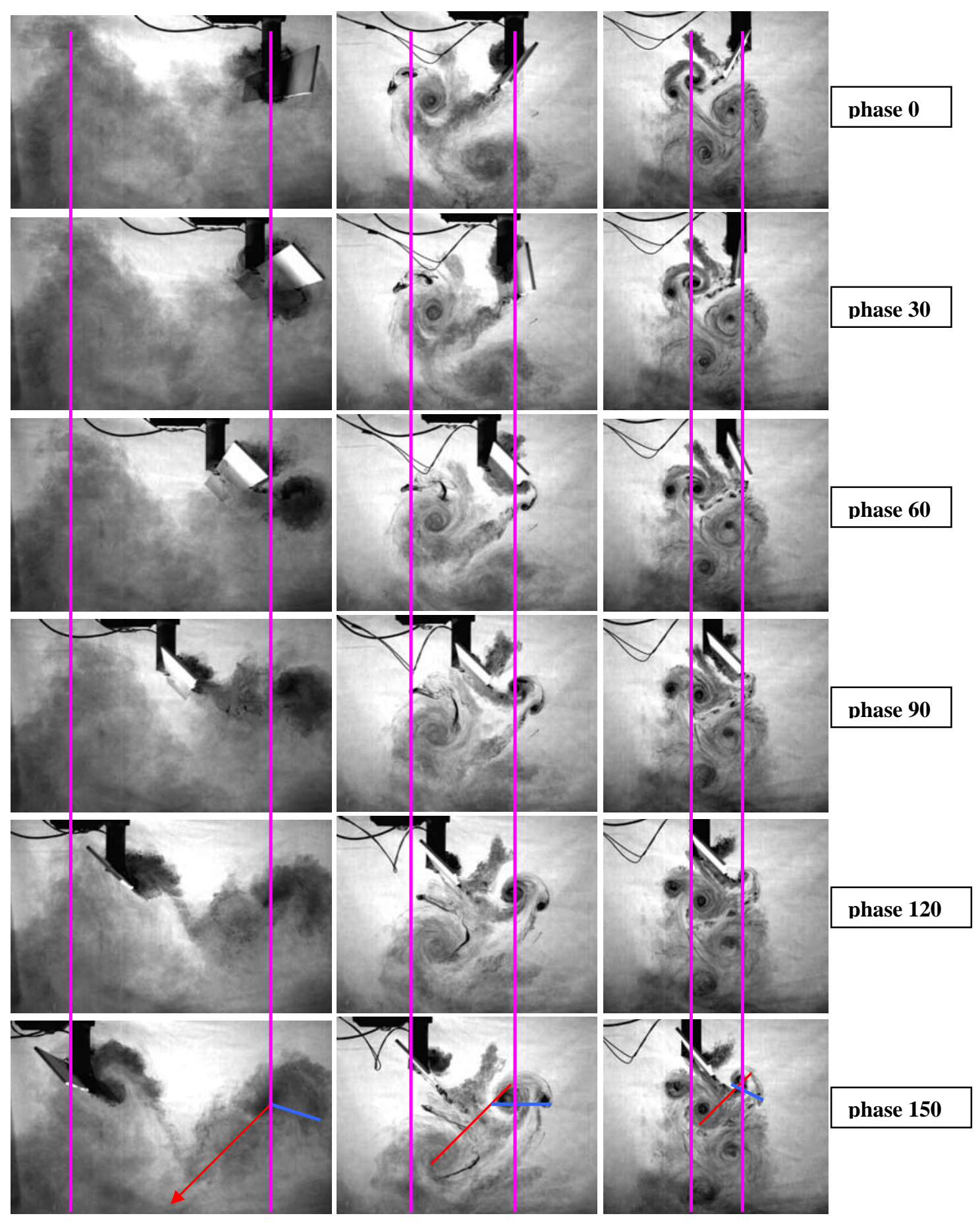




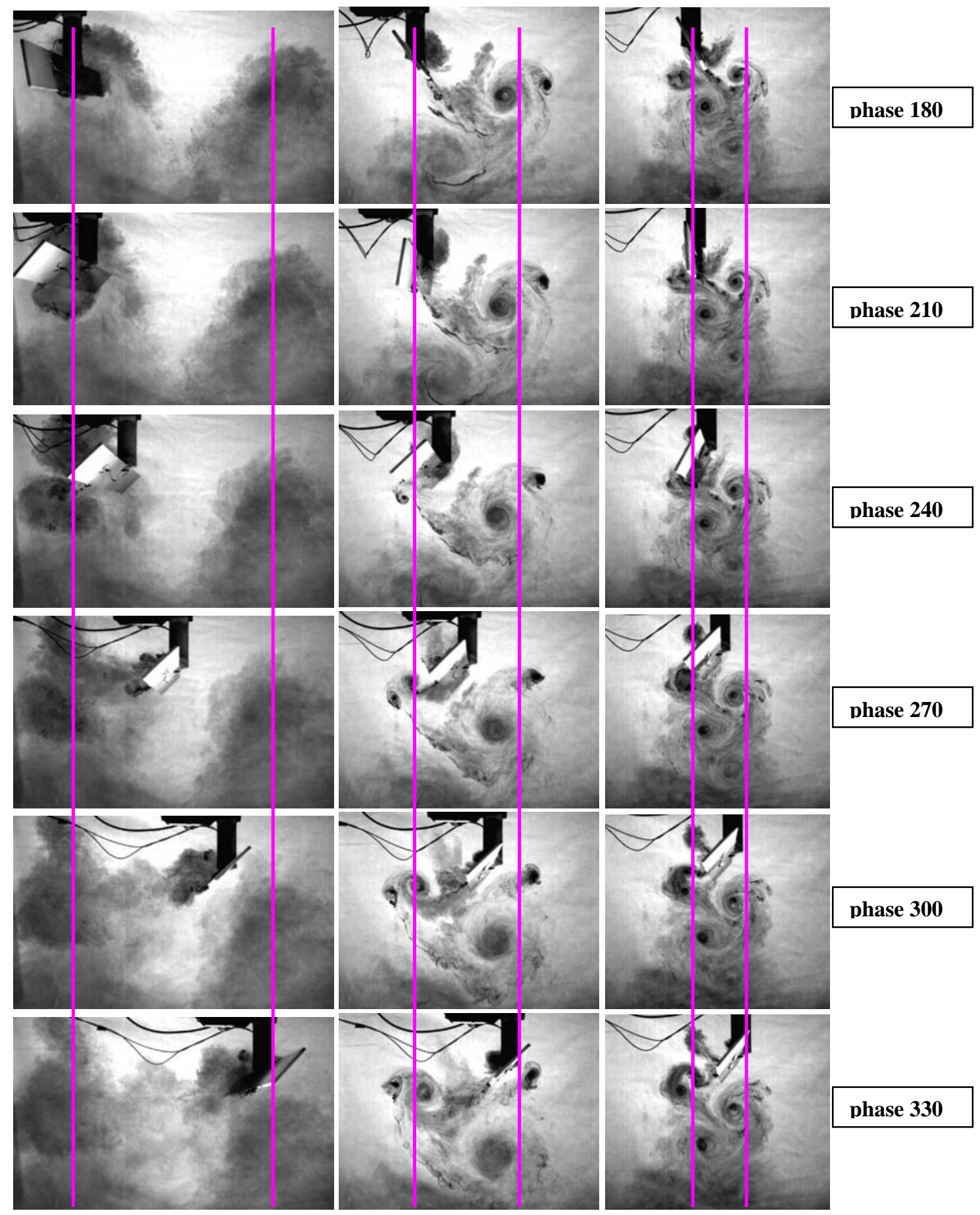

Figure 2. Dye injection snapshots for four sinusoidal fore-aft traverses of a wall-to-wall plate: $0.211 \mathrm{~Hz}, 3.1 \mathrm{c}$ amplitude (left column); $0.422 \mathrm{~Hz}, 1.55 \mathrm{c}$ amplitude $\left(2^{\text {nd }}\right.$ column); $0.844 \mathrm{~Hz}, 0.775 \mathrm{c}$ amplitude (right column). In going from top to bottom, with phase $0=$ right-most extreme of translation stroke, snapshots are at phase $=0,30,60,90,120,150,180,210,240,270,300$ and 330 degrees. Red and blue line segments (phase 180) attempt to correlate shed vortex pairs amongst the four cases. Nominal $\operatorname{Re}=6500$.

Phase "0" refers to the starboard extremum of the leading-edge fore-aft motion. All leading-edge motions are seen to be notionally sinusoidal. When normalized by stroke amplitude, the three translations 
become nearly coincident. The plate pivots between $\pm 45^{\circ}$ limits for all cases, with up to $2^{\circ}$ overshoot, due to slop in the hinge mechanism and elastic compliance in the hinge housing. If there were zero phase lag between the translation and rotation, the plate would be hanging vertically at translation phases of 0 and 180 degrees. Instead, the rotation lags the translation, akin to the "delayed rotation" 6 variant of normalhover. The longer the translation stroke to chord ratio, the lower the phase lag in rotation becomes.

A strong trailing edge vortex forms shortly after translation stroke reversal, but is quickly shed. This is common to all cases. Beyond that, the flowfield evolution varies case by case. For the largest amplitude ratio, a recirculating region forms on the lee side of the plate. Presently there is insufficient information to regard this as an LEV or not; but in any case, the entire lee side of the plate is covered by a concentration of dye convecting together with the plate, for approximately half of the translation semistroke. In the second half of the translation semi-stroke this structure is shed behind the lee side of the plate. Plate rotation at the opposite extremum of the translation semi-stroke results in a new TEV and eventually the formation of a new lee-side structure. The TEV, from either extremum of translational motion, is shed soon after formation, and persists near the vicinity of the translation endpoints. This structure participates in the dye mixing process and renders resolution of individual vortices difficult.

For the intermediate stroke to chord ratio, association of dye concentrations and evinced vortices is no longer so problematic. Instead of a trailing edge vortex at the extremum of each translation semistroke, there is a TEV pair; evidently one is due to rotation, and one to cessation of translation. An LEVlike structure forms soon after translation phase phi $=0$, but is shed, to become a sort of column-structure emanating from the plate's lee side. A new LEV forms and persists until the opposite translation halfstroke extremum, where it finally sheds and convects along the plate while the plate rotates, entering the wake. A new TEV pair forms, and the ensuing collection of vortices forms an alternating sequence into the wake below the plate. Conceivably a similar vortex train could be possible with the lowest frequency case, but there the large amplitude may be expected to run into blockage problems, because the end-toend extent of translation is now comparable to the distance from the plate TE to the tunnel test section bottom. A possible schematic rendering of the "axes" between shed vortices is speculatively noted by the red and blue lines for phase phi $=150$ in Figure 2.

For the highest frequency/lowest amplitude case, phase lag between translation and rotation is on the order of 45 degrees. The proliferation of vortices becomes too complicated to associated with plate kinematic events, other than to note that vortex appear to be shed in pairs.

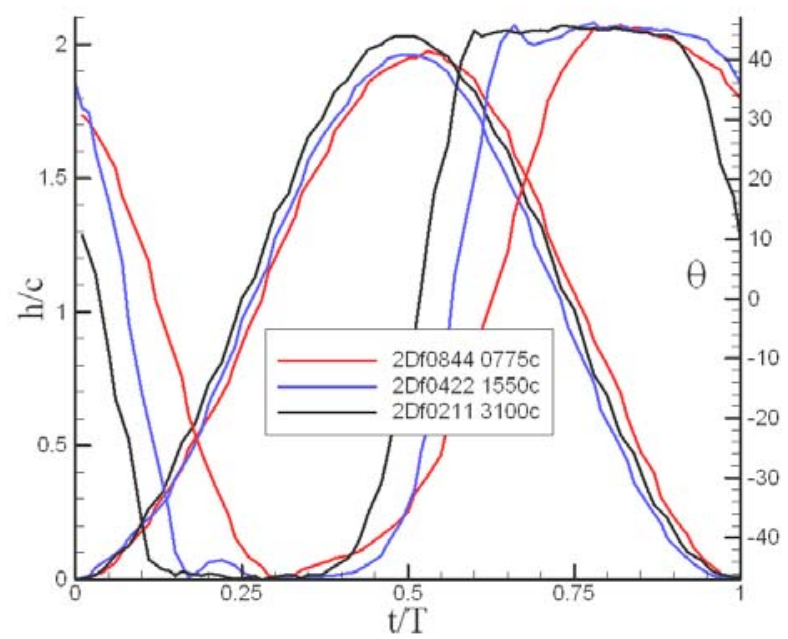

Figure 3. History of leading edge position and plate incidence angle over one stroke period, normalized by stroke amplitude and time for one period; wall-to-wall flat plate, cases as in Figure 2.

We next turn to parameter studies of thrust coefficient and normal-force coefficient for the various cases in Table 1. Thrust is taken to be in the vertical direction in the lab reference frame; that is, 
normal to the direction of leading edge fore-aft translation. Normal force is in the direction opposing the fore-aft translation. Thus, "thrust" is a kind of lift, while "normal force" becomes a drag.

Figure 4 gives a survey of thrust and normal force coefficients for a range of translation amplitudes, comparing the various frequencies in the same respective plot. This amounts to a Reynolds number survey for each translation amplitude. Across the Reynolds number range considered here, the aerodynamic force coefficients do not have a demonstrable Reynolds number dependency, provided that the translation speed for the lower Reynolds number cases is sufficiently high to produce a plate incidence angle history that comports with that of the higher-speed cases. That is, too slow of a motion will to "swing" the plate completely against its limiters. This is partially the case for $0.211 \mathrm{~Hz}, 3.1 \mathrm{c}$ amplitude, which shows a departure from the other 3.1c curves.
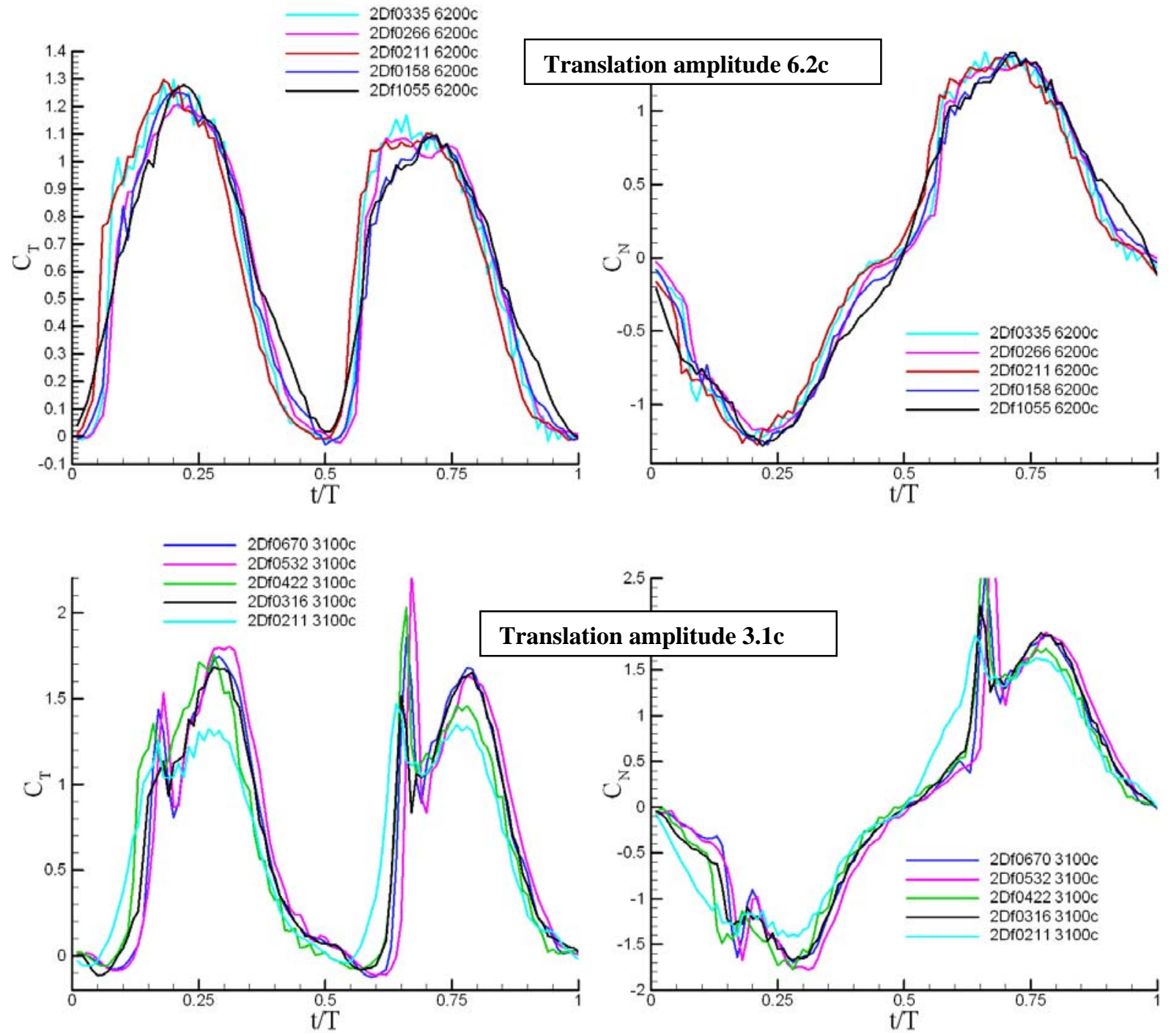

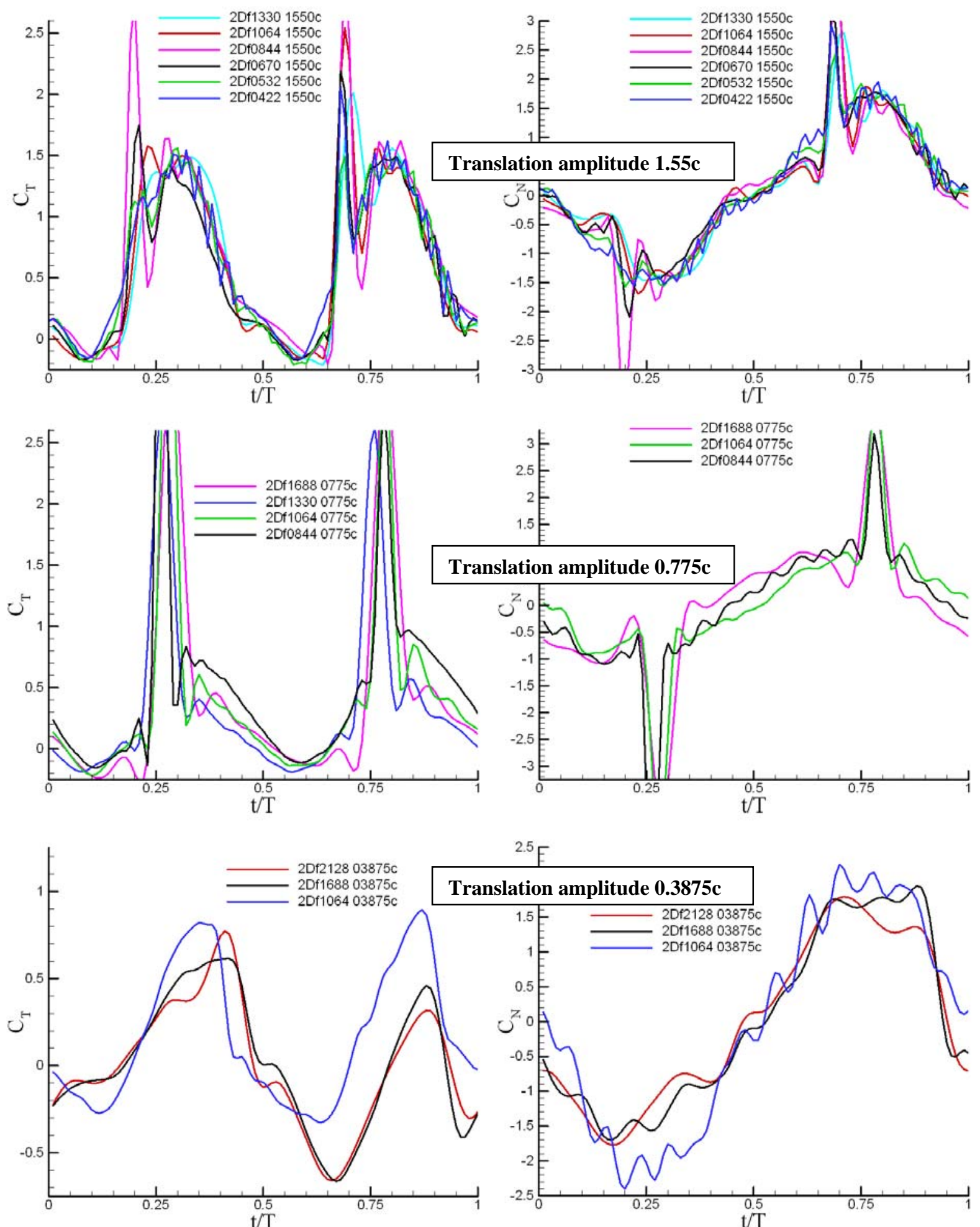

\section{${ }^{2.5} \mathrm{E}$}
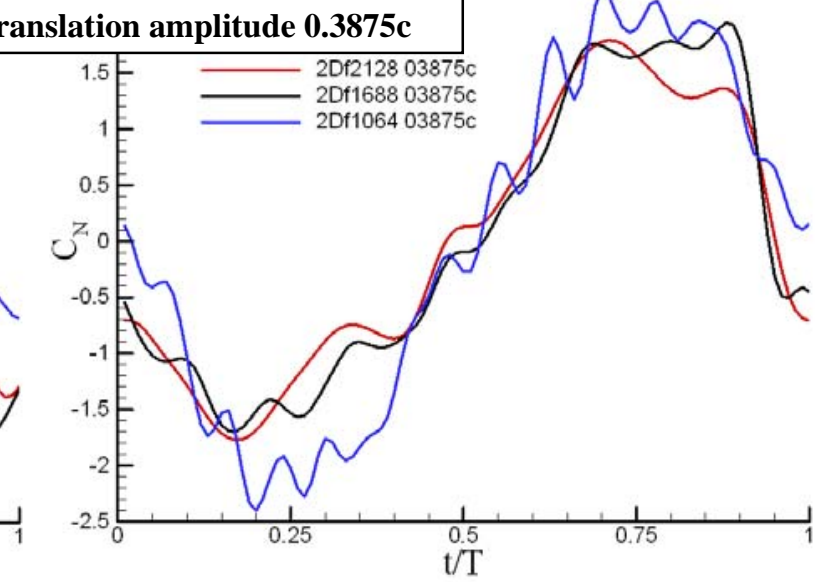

Figure 4. Thrust coefficient (left column) and normal force coefficient (right column) for various frequencies of translational oscillation of a $2 \mathrm{D}$ plate. Reading from top to bottom row, leading-edge translation amplitudes are 6.2c, 3.1c, 1.55c, $0.775 \mathrm{c}$ and $0.3875 \mathrm{c}$. 
The relative unimportance of Reynolds number means that the remaining defining parameter is stroke to chord ratio. Figure 5 compares various stroke to chord ratios while nominally keeping Reynolds number constant. For the larger stroke to chord ratios, thrust coefficient and normal force coefficient are roughly sinusoidal, with the latter of the same frequency as the leading edge translation, and the former at double the frequency. The principal departure from sinusoidal response is spikes at or just before $\mathrm{t} / \mathrm{T}=$ 0.25 and 0.75 . Spikes are due to the plate hitting against its pitch limiter, and not due to noncirculatory loads. Associating spikes in the force coefficients with the rotation of the plate, it is clear that lag between rotation and translation increases with decreasing stroke to chord ratio, as noted earlier.
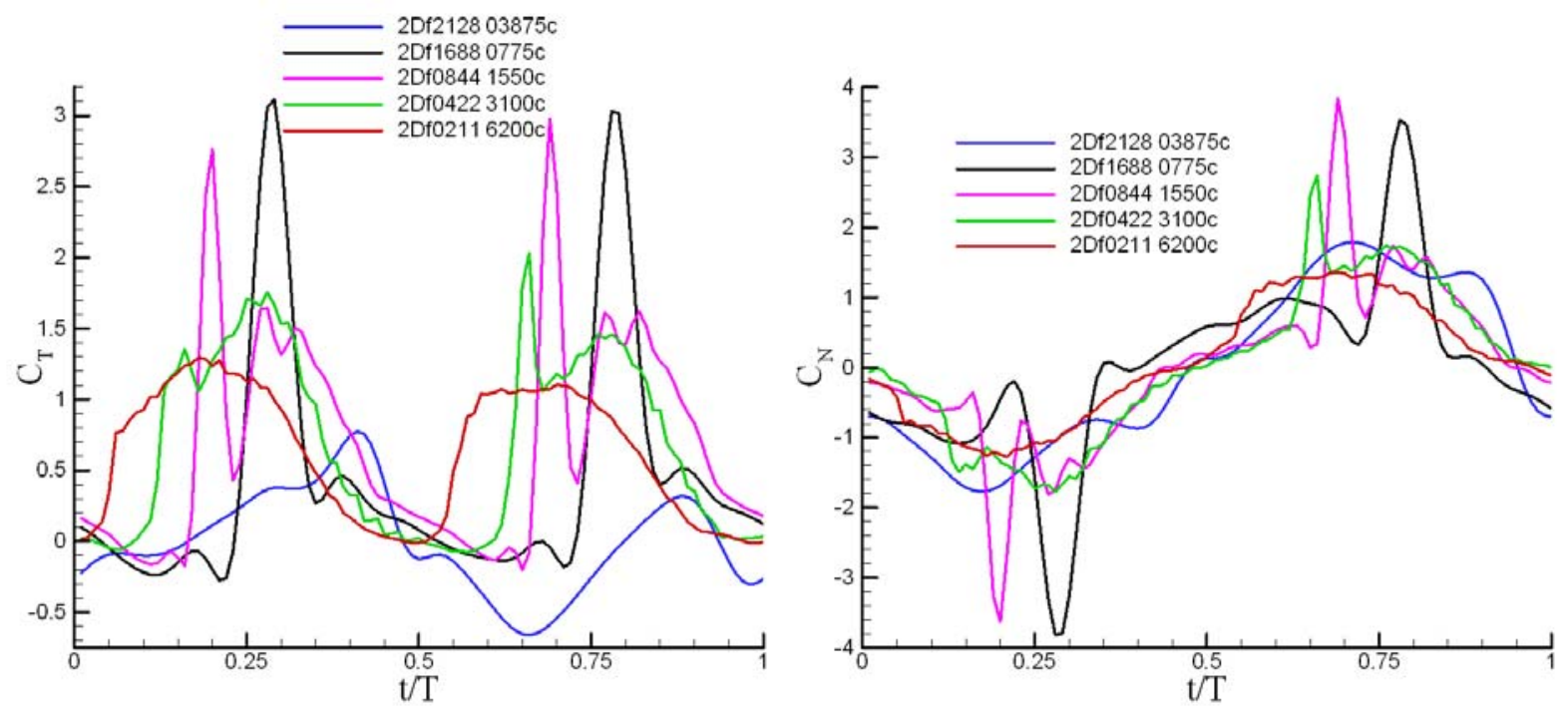

Figure 5. Thrust coefficient (left) and normal-force coefficient for a series of stroke to chord ratios. Nominal $\mathrm{Re}=6500$, except for the lowest stroke to chord ratio case.

The intermediate ranges of stroke to chord ratio $-1.55 \mathrm{c}$ and $3.1 \mathrm{c}$ - appear to give the highest thrust coefficient. Going to an even large ratio of $6.2 \mathrm{c}$ appears to slightly reduce thrust coefficient, though the difference is not large. Much clearer is attenuation of thrust when the stroke to chord ratio is small. For a ratio of $0.3875 \mathrm{c}$, the plate produces almost zero net thrust, essentially serving to mix the flowfield through a series of vortices that interact constructively and destructively. Normal force coefficient, on the other hand, does not appear to vary much amongst any of the examined cases, apart from the appearance of spikes when the plate hits its limiter.

\section{2. $A R=3.4$ plate with sinusoidal rectilinear fore-aft motion}

We next extend the previous section's results to a plate of finite aspect ratio. AR=3.4 was selected, following a proposed conceptual design by Doman et al. ${ }^{1}$ The principal question is whether and to what extent - the tip vortices produce a spanwise suction that leads to LEV stabilization, and therefore to a material difference in the evinced flow separation history, and further, in the force coefficient history.
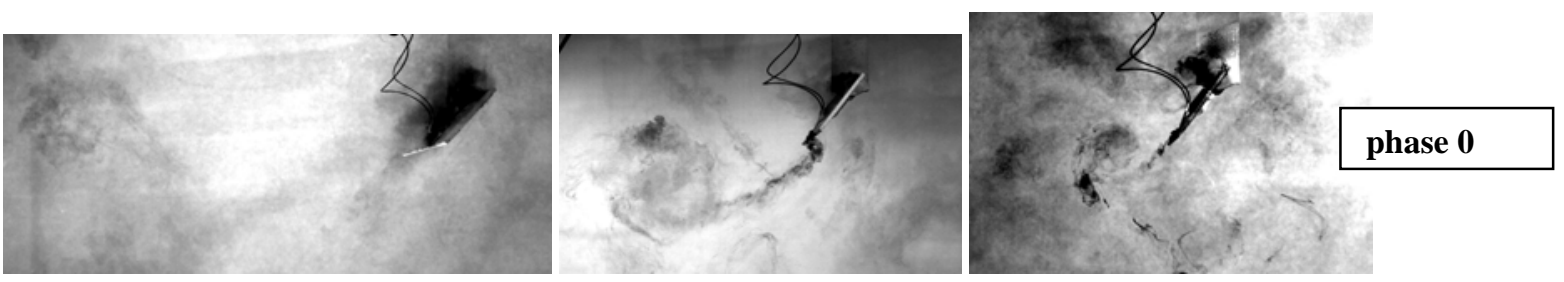

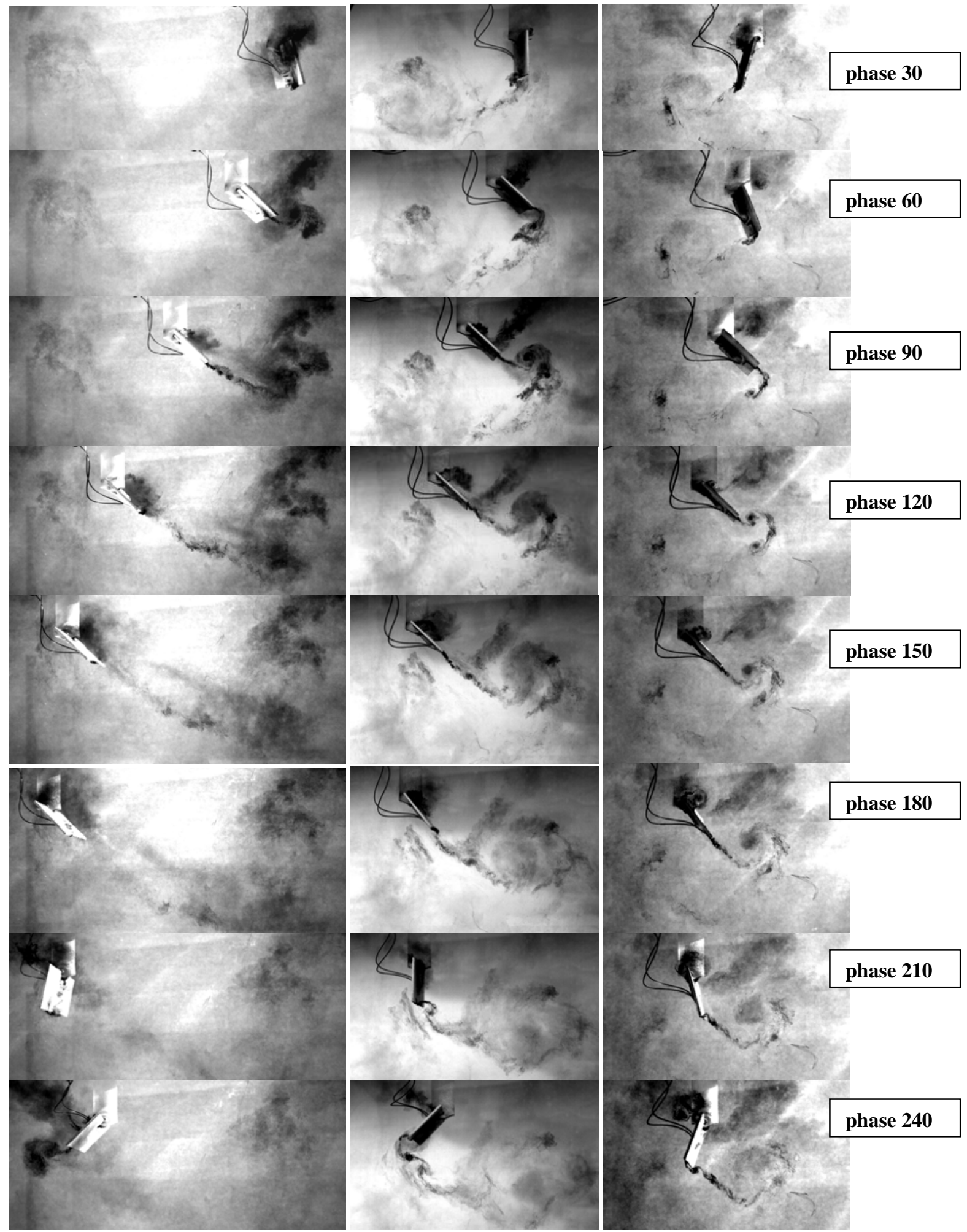

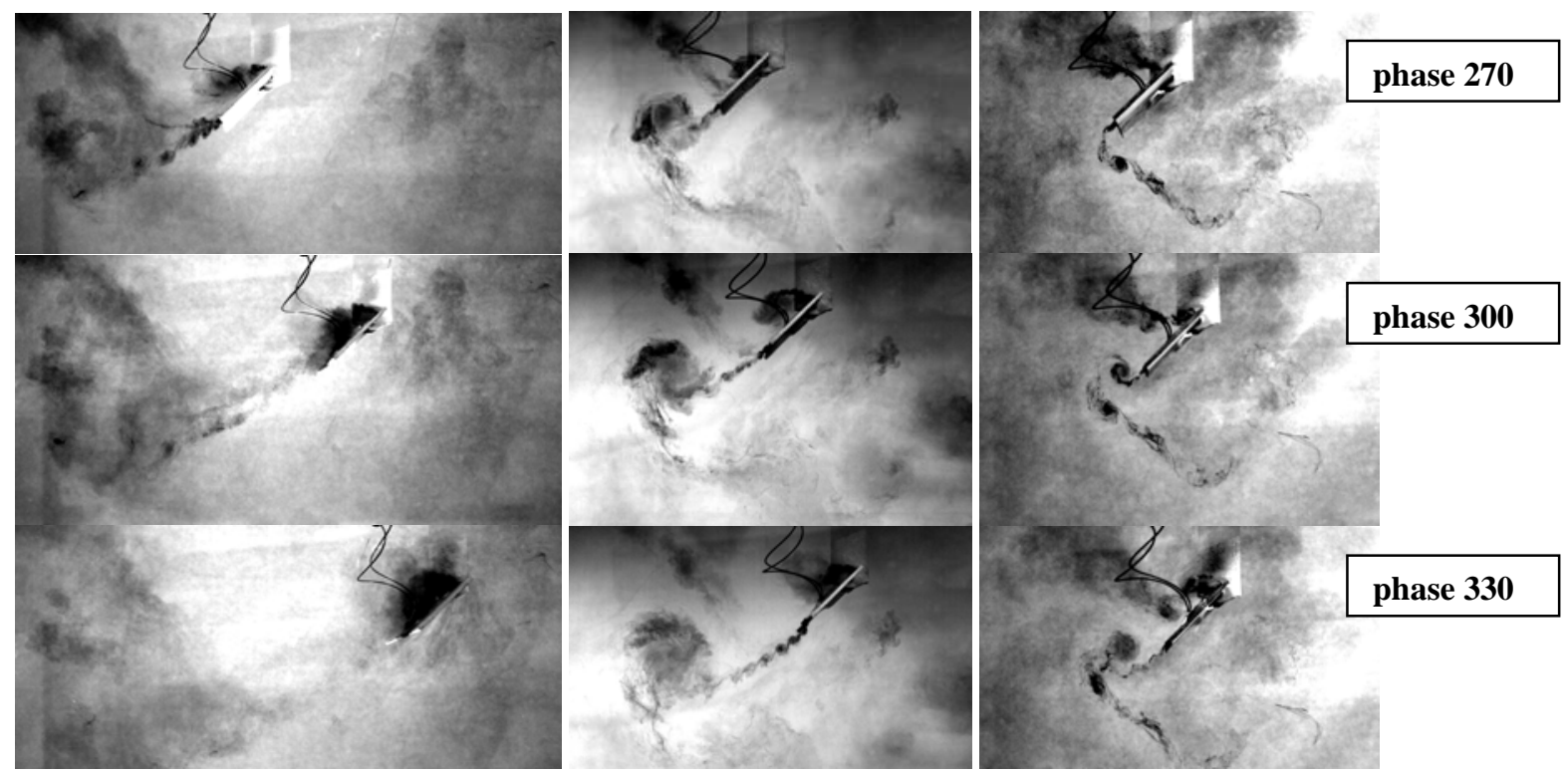

Figure 6. Dye injection snapshots for three sinusoidal fore-aft traverses of an aspect ratio 3.4 plate: $0.422 \mathrm{~Hz}$, 3.1c amplitude (left column); $0.844 \mathrm{~Hz}, 1.55 \mathrm{c}$ amplitude $\left(2^{\text {nd }}\right.$ column); and $1.688 \mathrm{~Hz}, 0.775 \mathrm{c}$ amplitude (right column). In going from top to bottom, with phase $0=$ right-most extreme of translation stroke, snapshots are at phase $=0,30,60,90,120,150,180,210,240,270,300$ and 330 degrees. Note that here the Reynolds number is twice that of the three data sets in Figure 2.

Dye injection results for a survey of frequency/amplitude, while keeping Reynolds number constant, are given in Figure 6. These are analogous to the 2D plate results in Figure 2, except that the Reynolds number here is twice as high (13000). As with the $2 \mathrm{D}$ case, dye is injected at the $3 / 4$-span location, at both the leading and trailing edge of the plate.

To render useful comparison with the 2D plate's results, we first assess whether the AR=3.4 plate's kinematics are similar. Figure 7 repeats the $2 \mathrm{D}$ plate results from Figure 3, and adds the $A R=3.4$ plate results from the dye injection in Figure 6.
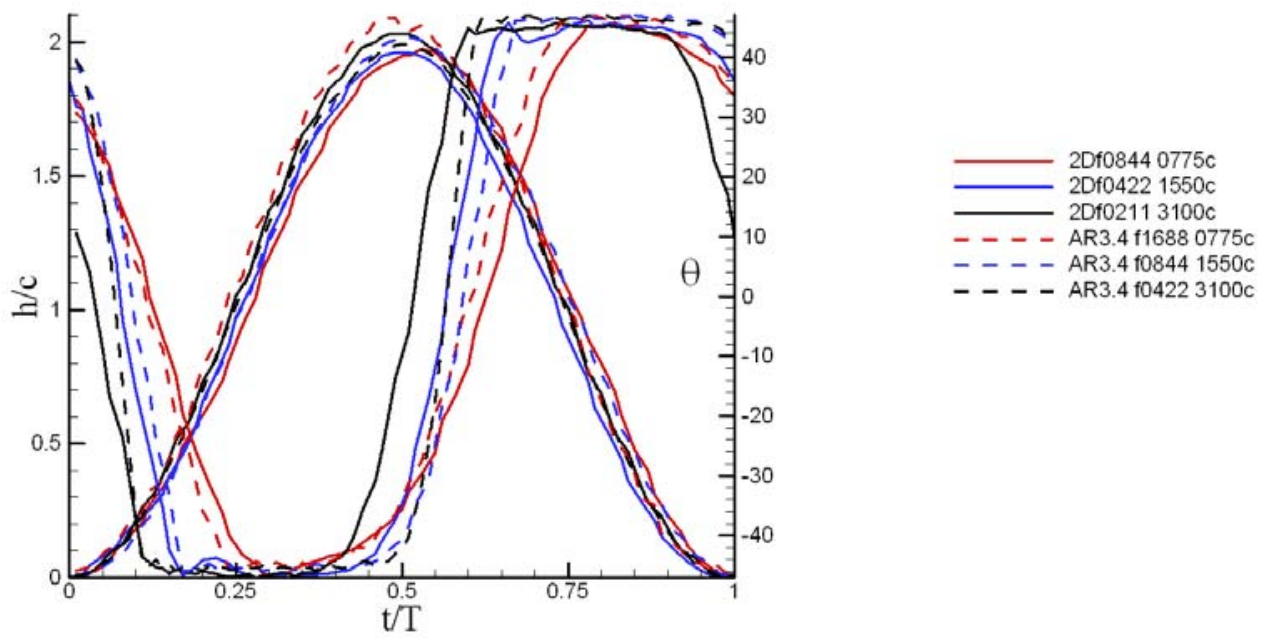

Figure 7. Comparison on $2 \mathrm{D}$ plate and $\mathrm{AR}=3.4$ plate motion kinematics for selected translating cases.

Discrepancies in the sinusoid describing the leading-edge motion history are primarily those of perspective and vibration of the model, due to slop in the hinge and consequent lateral motion of the plate. This was larger for the $\mathrm{AR}=3.4$ plate than for the 2D plate. Discrepancy between the two plates' 
incidence angle history is due to similar causes, and also to differences in hinge dynamics; the $\mathrm{AR}=3.4$ plate has only $37 \%$ of the mass of the 2D plate, and therefore less means to overcome friction in its leading-edge hinge. Nevertheless, in the bulk the two plates' respective motion histories are similar.

As with the 2D plate, we proceed with a survey of force coefficients for various stroke/chord amplitudes vs. motion frequency, or in other words, vs. Reynolds number (Figure 8). Coefficients are again referenced to peak translational speed and to the plate's area. Therefore, difference between the 2D and $\mathrm{AR}=3.4$ plate would be an effect of aspect ratio directly. But curiously, there is little difference between the respective counterparts in Figure 4 and Figure 8. This is confirmed in Figure 9, where the dashed lines ( $\mathrm{AR}=3.4$ plate) closely follow the solid lines (2D plate) for all of the stroke to chord ratios under consideration. The one notable discrepancy is in normal force coefficient for the smallest stroke to chord ratio, where presumably hinge dynamics play a larger role. Discrepancies in phase position of force coefficient spikes between the $2 \mathrm{D}$ and $\mathrm{AR}=3.4$ plates correlate in the respective discrepancies in plate incidence angle history in Figure 7, giving further evidence that the force coefficient spikes are indeed due to the plate hitting its incidence limiters. As with the 2D plate, thrust coefficient declines for small stroke to chord ratios, but normal force coefficient differs amongst the various stroke to chord ratios mainly just in the force spikes.
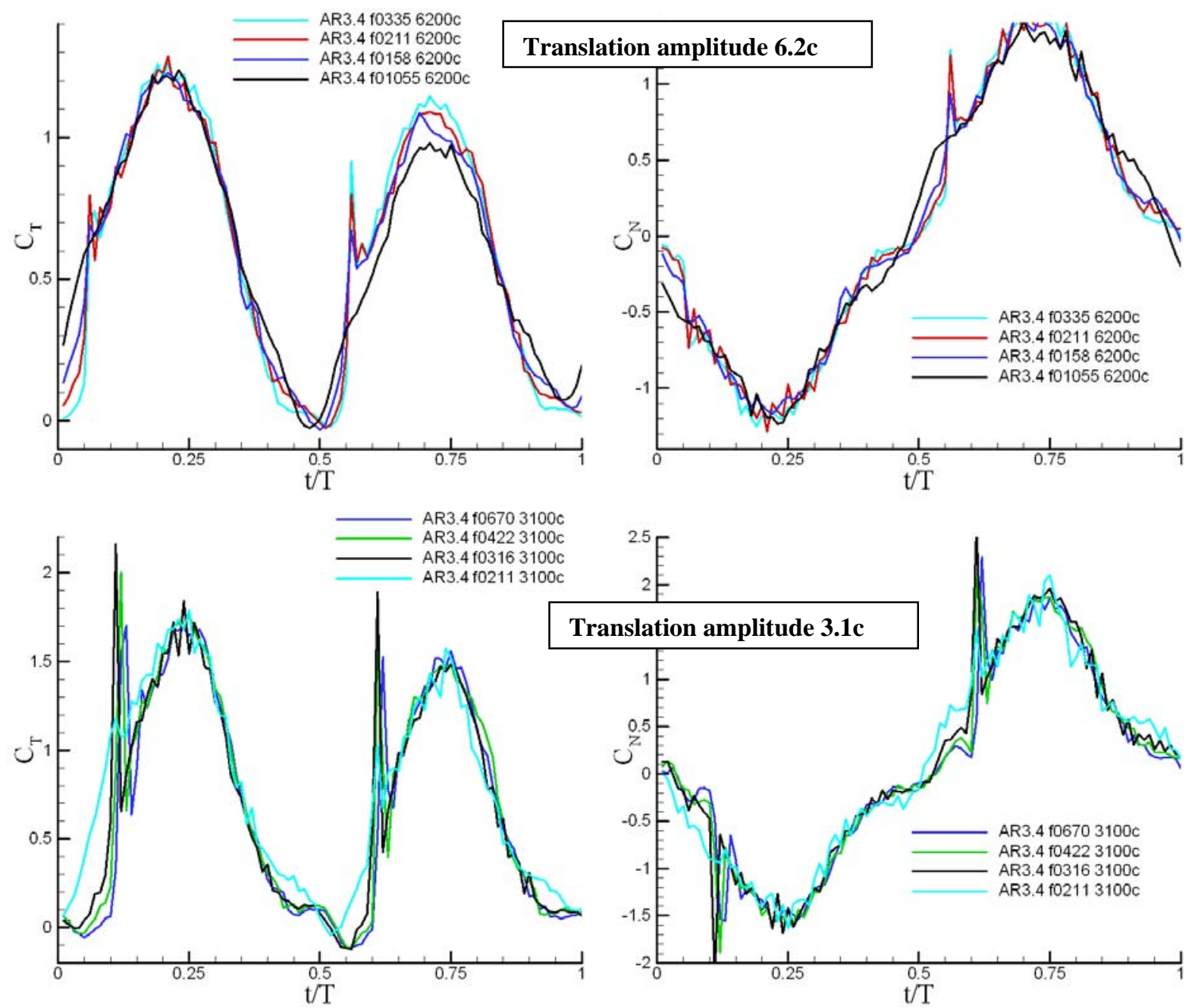

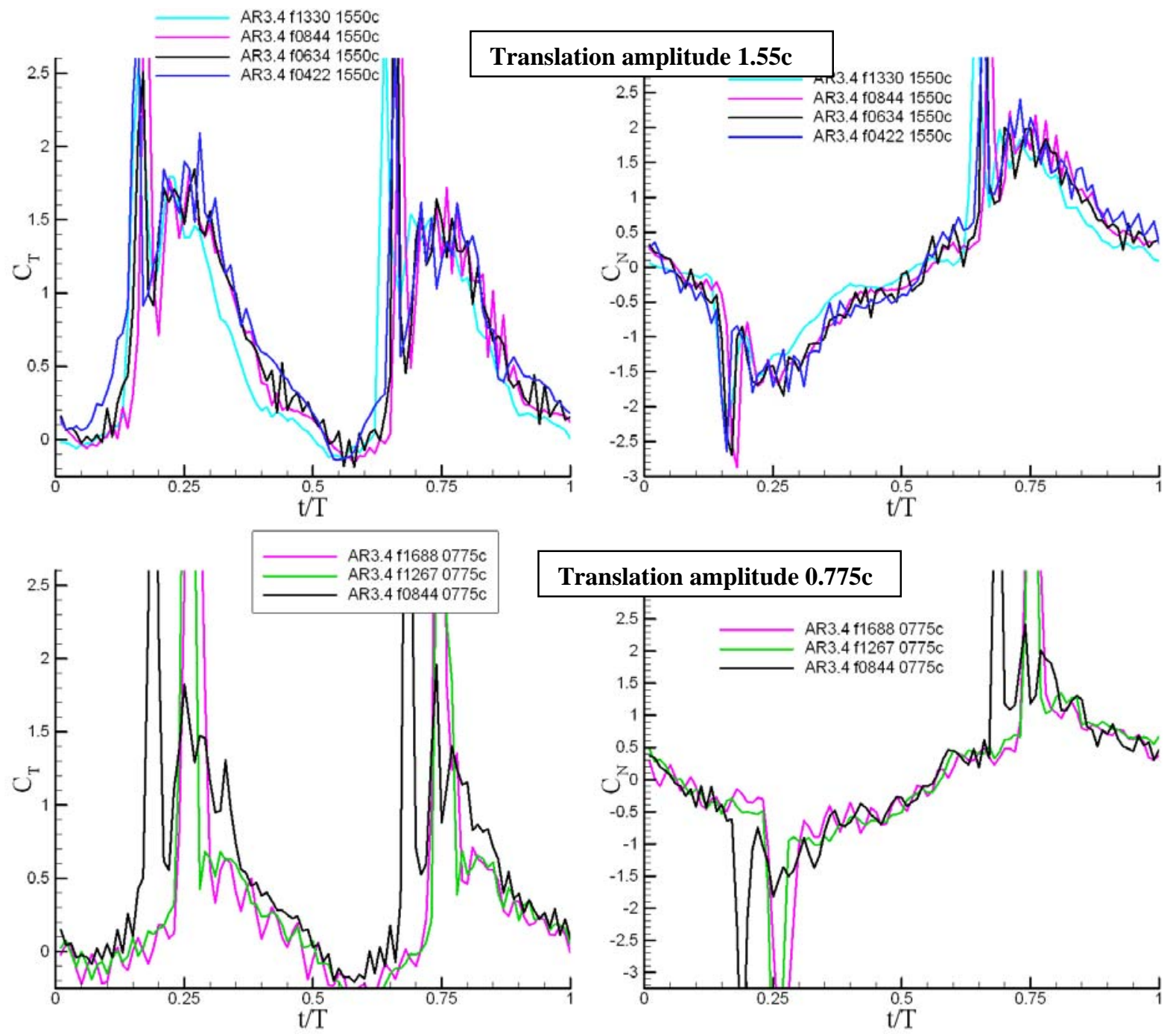

Figure 8. Thrust coefficient (left column) and normal force coefficient (right column) for various frequencies of translational oscillation of a $2 \mathrm{D}$ plate. Reading from top to bottom row, leading-edge translation amplitudes are $6.2 c, 3.1 c, 1.55 c$, and $0.775 c$.

It is also worth noting that in none of the examined cases was there a double-peak in thrust coefficient in any semi-stroke. That is, thrust is correlated - if not outright quasi-steady - with translational speed amplitude, and there is no evidence of a wake-capture phenomenon where there would be a second thrust peak due to plate's favorable interaction with a vortex shed during the previous halfstroke. 

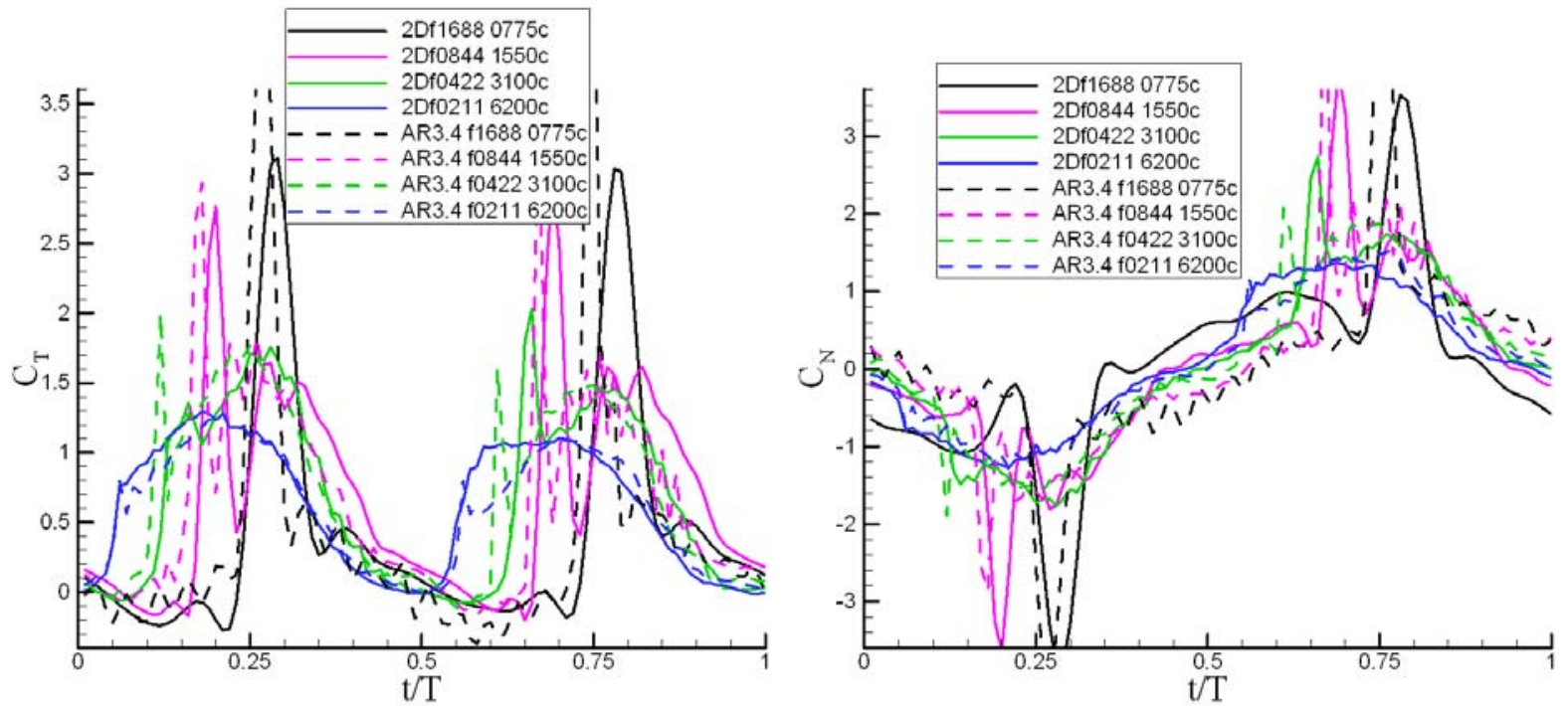

Figure 9. Comparison of thrust coefficient (left) and normal force coefficient (right) for the $2 \mathrm{D}$ and $\mathrm{AR}=3.4$ plates.

An alternative presentation of the force results is to consider the thrust and normal-force coefficients as functions of plate incidence angle. Figure 10 shows that force coefficients are nearly zero, in relative terms, while the plate is performing its rotational motion. That is, force coefficients at angles of incidence about $40^{\circ}$ absolute value are much larger than force coefficients for $-40^{\circ}<\theta<40^{\circ}$, implying that the rotational motion is less energetic than the translational, both in terms of useful force production (thrust) and loads necessary to produce the motion (normal force).
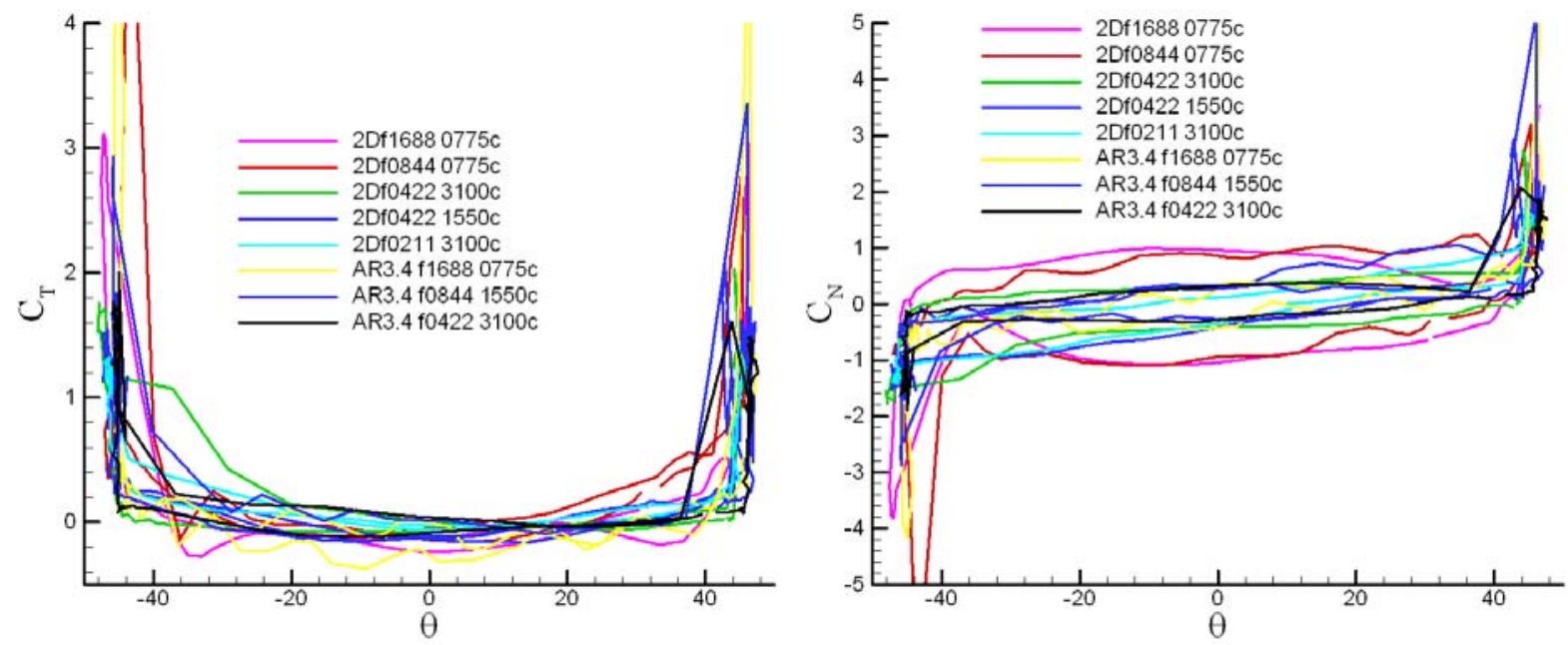

Figure 10. Thrust coefficient (left) and normal force coefficient (right) for all $2 \mathrm{D}$ and $\mathrm{AR}=3.4$ translating cases with recorded kinematics, plotted vs. plate incidence angle, instead of phase of translational motion.

Intuitively, high force coefficients at the extrema of plate incidence angle likely occur because the dynamic pressure is higher at the translation semi-stroke midpoint than during the rotation. Because the force coefficients are normalized by peak translational velocity, and not by a running time-history of velocity, the "coefficient" is essentially a dimensional force. That is, coefficients per se may be large during rotation, but when multiplied by the local dynamic pressure and divided by the peak dynamic pressured, one obtains a small coefficient. The practical consequence of the aerodynamic force coefficients reaching relatively large values at $|\theta|>40^{\circ}$, and relatively small values elsewhere, is substantiation of the simplifying assumptions useful for flapping-wing design. In particular, Doman et 
al. ${ }^{1}$ assumed that aerodynamic force can be taken as zero over the portion of the flapping stroke where the plate executes its rotation. To a first approximation, this is supported by the results of Figure 10.

\section{3. $A R=3.4$ plate with sinusoidal waving rotary motion}

The previous cases were limited to rectilinear motion, where every spanwise station had the same displacement history. The "waving" motion is more akin to flapping proper, where there is a spanwise variation in displacement, proportional to the distance from the spanwise station in question, to the pivot point. The pivot point is here taken as $0.5 \mathrm{c}$ onboard of the inner wingtip.

Dye injection results for the $\operatorname{Re} \sim 13,000(\mathrm{f}=0.422 \mathrm{~Hz}$ ) waving plate are given in Figure 11. As for the translating cases discussed above, snapshots are at every 30 degrees of the imposed motion. The directly relevant translating cases, based on tip speed, are the stroke to chord ratio of 3.1c.

Buoyancy of the plate is important in the waving motion, as a plate heavier or lighter than water will be biased towards one extreme of the angle of attack limiter for cases where dynamic pressure from the motion is insufficient to overcome the non-neutral buoyancy. While the plate is approximately neutrally buoyant, absorption of water from protracted submersion eventually makes the plate heavier than water. For this reason, the low frequency motions have a different angle of attack history than the higher-frequency motions, which in general collapse atop one another. This effect was reported earlier for the translating plates, but buoyancy exacerbates it for the waving plate. Unfortunately, the higher the physical frequency of motion, the more difficult it is to obtain dye injection images, because the faster motion produces more diffusion and less apparent cohesiveness of dye concentration.
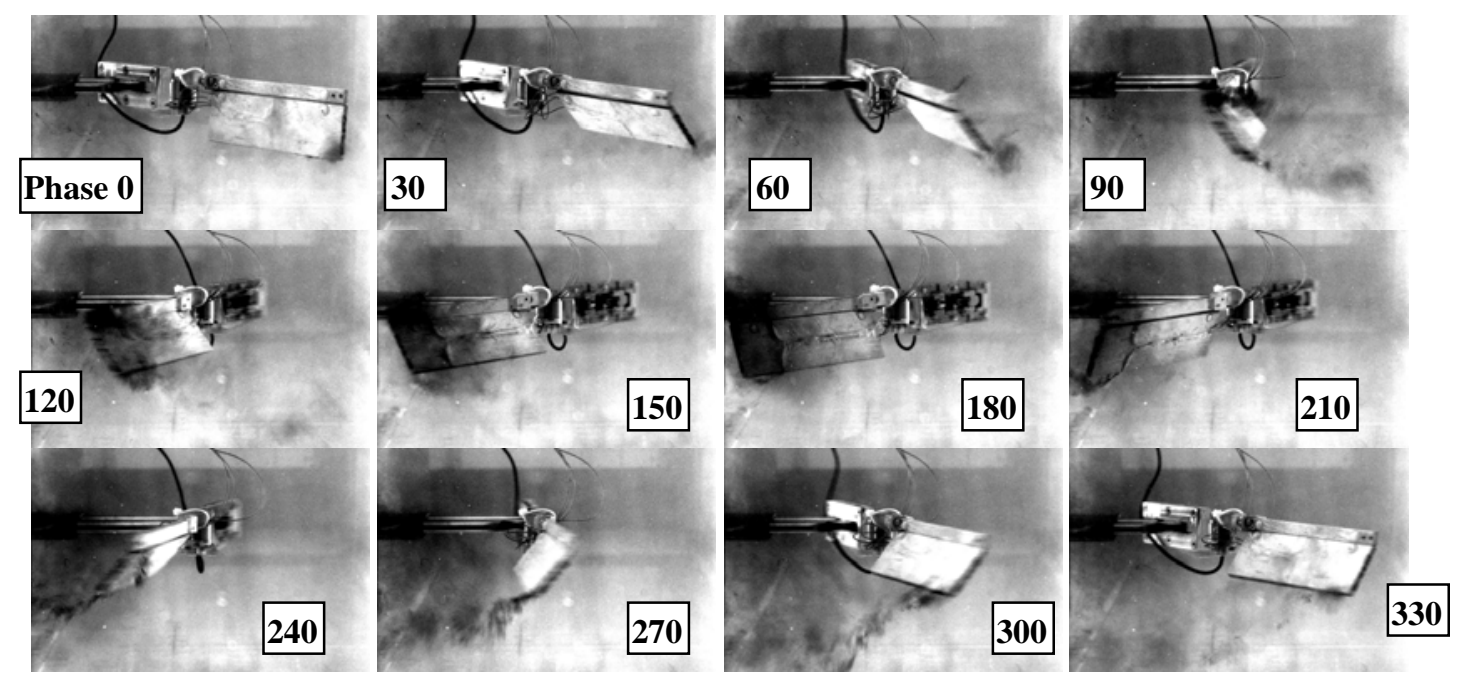

Figure 11. Flow visualization for waving $A R=3.4$ plate, $f=0.422 \mathrm{~Hz}, 90$-degree stroke amplitude. Reynolds number based on maximum tip speed is 13000 . Snapshots are in phases of motion of phi $=0,30,60, \ldots, 330$, in reading across the first row, then the second, and finally the bottom row.

All three families of motion cases $-2 \mathrm{D}$ and $\mathrm{AR}=3.4$ translating plates, and the $\mathrm{AR}=3.4$ waving plate - have a system of LE and TE vortices. TEVs in particular are similar, in forming during the plate's rotation, shedding immediately, and trailing behind the trailing edge during the translation phase of each half-stroke, connected to the TE by a train of discrete small vortices (shear layer roll-up). But the waving plate has the important difference from the other cases of a strong spanwise flow, as expected from the spanwise pressure gradient due to the outboard wingtip moving much faster than the inboard wingtip. Though Figure 11 is inconclusive owing to the poor image quality, it is not demonstrably the case that this spanwise flow or spanwise pressure gradient results in any greater prevalence of LEV or TEV attachment than in the translating cases. It remains to consider whether there is an effect on the aerodynamic force coefficients. 
Force coefficients for the waving plate are given in Figure 12. The motion amplitude in Figure 12 is designed to match the 3.1c stroke of the translating cases. Force coefficients are normalized using the peak speed of the leading edge at the $70 \%$ span location. The lowest frequency case, $\mathrm{f}=0.211 \mathrm{~Hz}$, departs markedly from the others, and its thrust coefficient history on the fore-stroke differs considerably from that of the back-stroke. This is due to buoyancy effects. Buoyancy is still somewhat observable in the $\mathrm{f}=0.422 \mathrm{~Hz}$ case, but is no longer a concern for $\mathrm{f}=0.532 \mathrm{~Hz}$ and above, whence the higherfrequency cases have mutually very similar force histories.
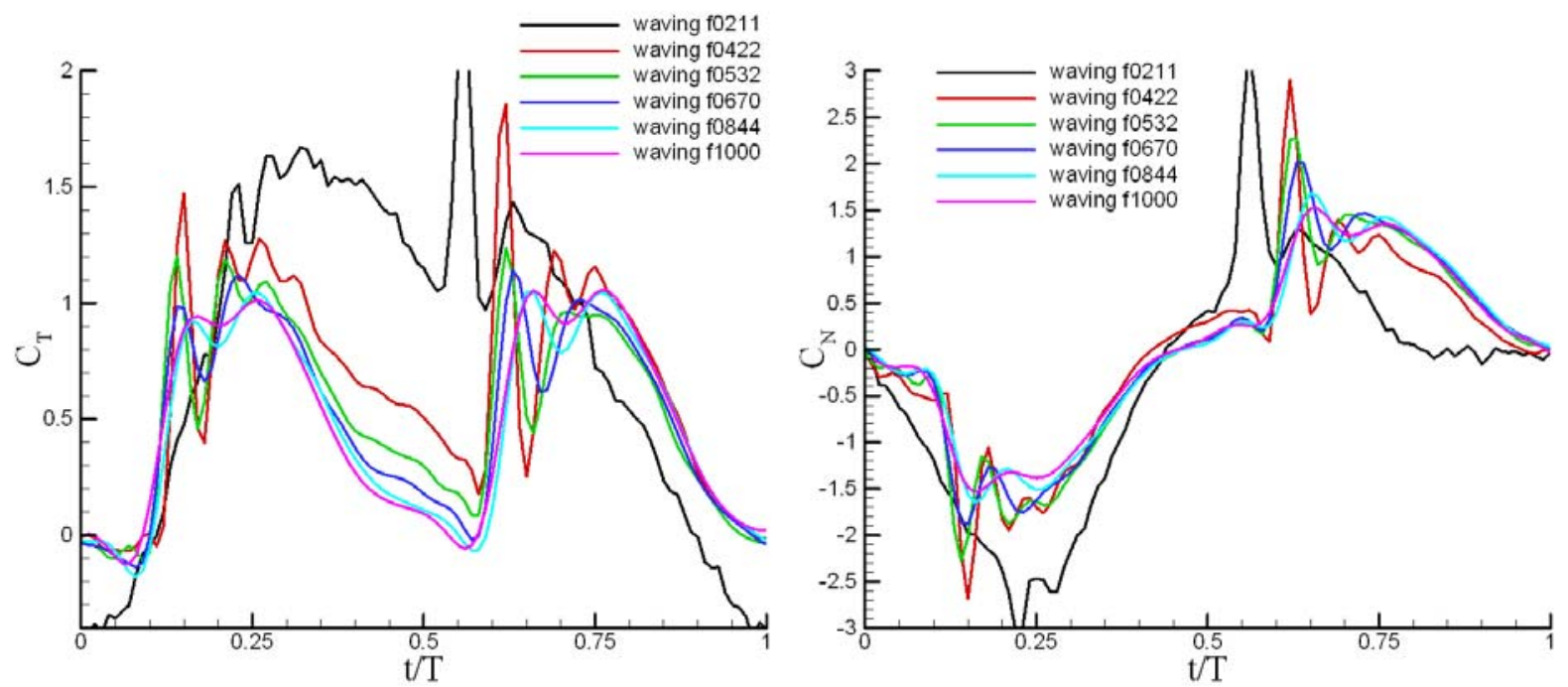

Figure 12. Thrust coefficient (left) and normal force coefficient (right) for the waving AR=3.4 plate.

Figure 13 compares the $2 \mathrm{D}$ translating plate, $\mathrm{AR}=3.4$ translating plate and $\mathrm{AR}=3.4$ waving plate, with motion frequency high enough such that buoyancy effects and hinge dynamics are of secondary concern $(f=6.70 \mathrm{~Hz})$. Normal force coefficient for the three cases is essentially identical. Thrust coefficient for the waving plate is lower at phases of waving motion where the tip speed is near maximum - that is, $0.15<\mathrm{t} / \mathrm{T}<0.35$ and $0.65<\mathrm{t} / \mathrm{T}<0.85$.
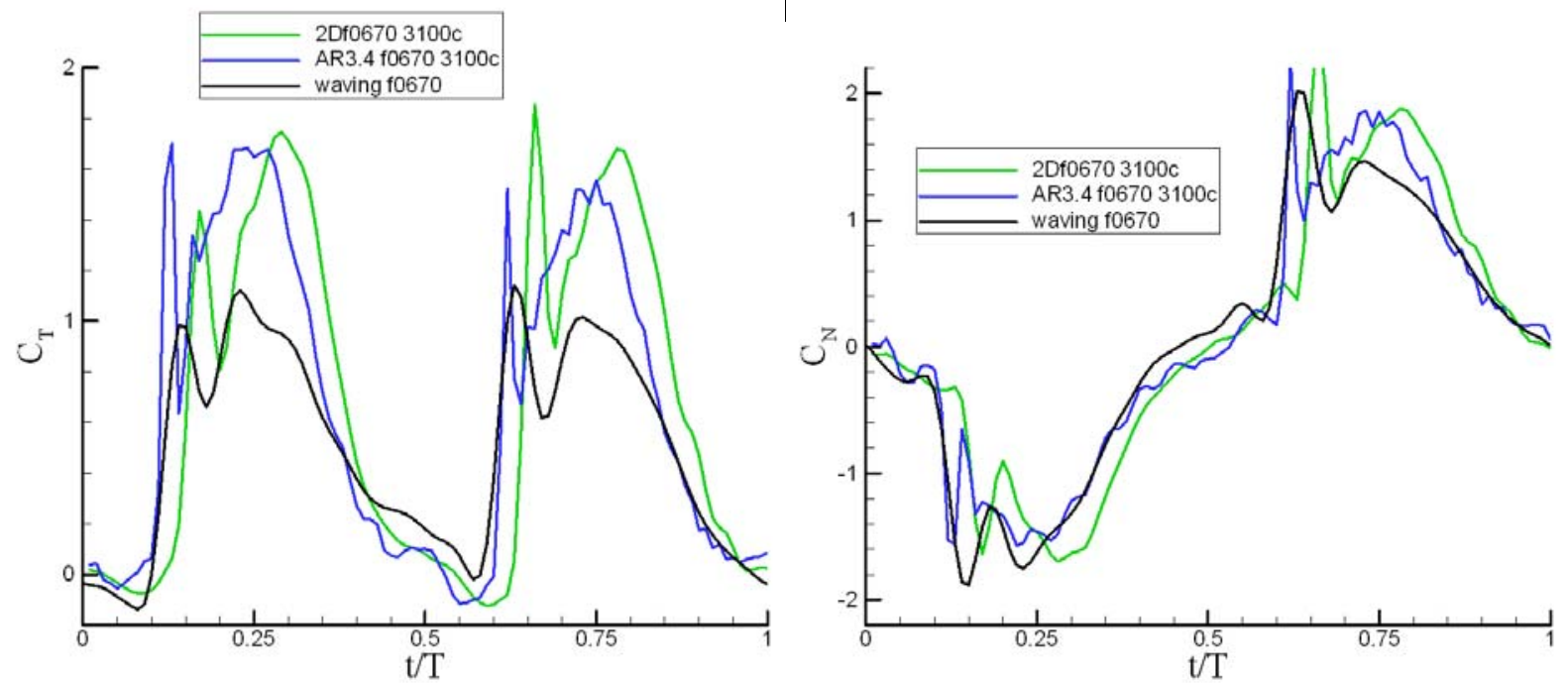

Figure 13. Comparison between the 2D translating plate, $A R=3.4$ translating plate, and $A R=3.4$ waving plate, with matched ratios of tip displacement amplitude to chord, and frequency $f=0.422 \mathrm{~Hz}$. 


\section{Conclusion}

A nominally 2D flat plate and aspect ratio 3.4 flat plate were subjected to prescribed sinusoidal translation of their leading edge, while the plate itself was free to pivot between $\pm 45^{\circ}$ incidence limiters. The $\mathrm{AR}=3.4$ plate was also studied in a flapping or waving motion, with prescribed sinusoidal pivot of the leading edge 0.5 chords outboard of one wingtip, with the plate again free to pivot between $\pm 45^{\circ}$ incidence limiters as it waved. Stroke to chord ratio was found to be the main factor affecting thrust production, with small stroke to chord ratios evincing the lowest thrust. Evidently, it is the translationdominated phase of motion, where the plate rests against its limiter, that is most responsible for aerodynamic force. All motions evince a shedding of a trailing edge vortex associated with the plate's rotation near the end of each imposed-motion semi-stroke. For the small stroke-to-chord ratio motions, the vortex shedding phenomena are complex, and are best summarized as a train of vortices of opposite sign coalescing into pairs, with the net effect of attenuating thrust, while generally not affecting the aerodynamic force resisting the imposed motion (here called the normal force). Reynolds number, in the range of 5000-20,000 for translation and 5000-30,000 for waving, was found to be relatively unimportant, provided that the resulting dynamic pressure was large enough to send the plate against its limiter, and to overcome effects of the plate's buoyancy in water. All three families of cases produced essentially the same normal force coefficient history, and similar thrust coefficient history, suggesting that neither aspect ratio nor the distinction between rectilinear and nonrectilinear motion makes large difference in thrust production. This implies that spanwise pressure gradients, however important in influencing vortex production, are of secondary importance in influencing integrated aerodynamic force.

\section{References}

\footnotetext{
${ }^{1}$ Doman, D., Oppenheimer, M., and Sigthorsson, D. "Dynamics and Control of a Minimally Actuated Biomimetic Hypersonic Vehicle: Part 1 - Aerodynamic Model”. AIAA 2009-6160.

2 Wood, R.J. "The First Takeoff of a Biologically Inspired At-Scale Robotic Insect". IEEE Transactions on Robotics, Vol. 24, No. 2., pp. 341-347, 2007.

${ }^{3}$ Milano, M., and Gharib, M. "Uncovering the Physics of Flapping Flat Plates with Artificial Evolution". J. Fluid Mech., Vol. 234, pp. 403-409, 2005.

${ }^{4}$ Kurtulus, D.F., David, L., Farcy, A., and Alemdaroglu, N. "A Parametric Study with Laser Sheet Visualization for an Unsteady Flapping Motion”. AIAA 2006-3917.

${ }^{5}$ Trizila, P.C., Kang, C.-K., Visbal, M.R., and Shyy, W. "Unsteady Fluid Physics and Surrogate Modeling of Low Reynolds Number, Flapping Airfoils". AIAA 2008-3821.

${ }^{6}$ Freymuth, P. "Thrust Generation by an Airfoil in Hover Modes". Experiments in Fluids, Vol. 9, pp. 17-24, 1990.

${ }^{7}$ Babinsky, H., and Jones, A.R. "Unsteady Lift Generation on Sliding and Rotating Flat Plate Wings." AIAA 20093689.

${ }^{8}$ O1, M., Bernal, L., Kang, C.-K., and Shyy, W. "Shallow and Deep Dynamic Stall for Flapping Low Reynolds Number Airfoils". Experiments in Fluids, Vol. 46, Issue 5, pp. 883-901, May 2009.

${ }^{9}$ Ol, M., McAuliffe, B. R., Hanff, E. S., Scholz, U., Kaehler, Ch., "Comparison of Laminar Separation Bubble Measurements on a Low Reynolds Number Airfoil in Three Facilities", AIAA 2005-5149.

${ }^{10}$ Ol, M.V. "Vortical Structures in High Frequency Pitch and Plunge at Low Reynolds Number". AIAA-20074233.
} 